\title{
THE IMPACT OF METALLICITY ON THE RATE OF TYPE Ia SUPERNOVAE
}

\author{
Matthew D. Kistler ${ }^{1,2,6}$, K. Z. Stanek ${ }^{3,4}$, Christopher S. KochaneK ${ }^{3,4}$, José L. Prieto ${ }^{5,7}$, and Todd A. Thompson $3,4,8$ \\ ${ }^{1}$ California Institute of Technology, Mail Code 350-17, Pasadena, CA 91125, USA \\ 2 Department of Physics, University of California, Berkeley and Lawrence Berkeley National Laboratory, Berkeley, CA 94720, USA \\ ${ }^{3}$ Center for Cosmology \& Astro-Particle Physics, The Ohio State University, Columbus, OH 43210, USA \\ ${ }^{4}$ Department of Astronomy, The Ohio State University, Columbus, OH 43210, USA \\ ${ }^{5}$ Carnegie Observatories, 813 Santa Barbara Street, Pasadena, CA 91101, USA \\ Received 2012 August 3; accepted 2013 April 30; published 2013 May 28
}

\begin{abstract}
The metallicity of a star strongly affects both its evolution and the properties of the stellar remnant that results from its demise. It is generally accepted that stars with initial masses below $\sim 8 M_{\odot}$ leave behind white dwarfs and that some sub-population of these lead to Type Ia supernovae (SNe Ia). However, it is often tacitly assumed that metallicity has no effect on the rate of SNe Ia. We propose that a consequence of the effects of metallicity is to significantly increase the SN Ia rate in lower-metallicity galaxies, in contrast to previous expectations. This is because lower-metallicity stars leave behind higher-mass white dwarfs, which should be easier to bring to explosion. We first model SN Ia rates in relation to galaxy masses and ages alone, finding that the elevation in the rate of $\mathrm{SNe}$ Ia in lower-mass galaxies measured by Lick Observatory SN Search is readily explained. However, we then see that models incorporating this effect of metallicity agree just as well. Using the same parameters to estimate the cosmic SN Ia rate, we again find good agreement with data up to $z \approx 2$. We suggest that this degeneracy warrants more detailed examination of host galaxy metallicities. We discuss additional implications, including for hosts of high- $z$ SNe Ia, the SN Ia delay time distribution, super-Chandrasekhar SNe, and cosmology.
\end{abstract}

Key words: binaries: close - supernovae: general - white dwarfs

Online-only material: color figures

\section{INTRODUCTION}

The end result of the evolution of stars that produce white dwarfs (WDs) is often a Type Ia supernova (SN Ia) explosion, through a single-degenerate channel (e.g., Whelan \& Iben 1973), double-degenerate channel (e.g., Iben \& Tutukov 1984; Webbink 1984), or both. Since stellar evolution is obviously affected by metallicity, there is no a priori reason why the rate of SNe Ia should not significantly depend on metallicity. From a theoretical standpoint, a preference for high metallicity was proposed by Kobayashi et al. (1998), whose single-degenerate model required a minimum metallicity of $\sim 0.1 Z_{\odot}$ in order to produce SNe Ia. A similar preference for higher metallicity was seen in the single degenerate models of Langer et al. (2000). However, the strong predictions offered by these models, such as no SNe Ia in dwarf galaxies and the outskirts of spirals, were not confirmed observationally (e.g., Prieto et al. 2008). The viability of the single-degenerate channel to produce the majority of SNe Ia has been debated from both observational (e.g., Leonard 2007; Simon et al. 2009; Gilfanov \& Bogdan 2010) and theoretical viewpoints (Ruiter et al. 2009; Kasen et al. 2009; Hachisu et al. 2010), as has the double degenerate scenario (Pakmor et al. 2010, 2012; Fryer et al. 2010; Shen et al. 2012; Dan et al. 2012; Zhu et al. 2013).

From the viewpoint of stellar evolution, we expect an opposite sign for the dependence of the rate of SNe Ia on metallicity. Stars of lower metallicity at a given mass generally produce more massive WDs according to stellar evolution calculations (e.g., Umeda et al. 1999; Marigo \& Girardi 2007; Meng et al.

\footnotetext{
6 Einstein Fellow.

7 Hubble, Carnegie-Princeton Fellow.

8 Alfred P. Sloan Fellow.
}

2008), which should make it easier for them to reach the Chandrasekhar mass and explode. For example, according to Marigo \& Girardi (2007), a single star with an initial mass of $3 M_{\odot}$ will leave behind a $\simeq 0.7 M_{\odot}$ WD when evolved at solar metallicity $\left(Z_{\odot}=0.019\right)$, while a star with the same initial mass at much lower metallicity $(Z=0.001)$ will leave behind a $>0.8 M_{\odot}$ WD. Due to the steepness of the stellar initial mass function (IMF), this leads to a larger number of SN Ia progenitors. Obviously, producing an SN Ia explosion is a more complicated process than just evolving single stars (for example, see the discussion of common envelope phase treatment in Ruiter et al. 2009). However, the observed rate of SNe Ia implies that a large fraction $(\sim 2 \%-40 \%)$ of all $3 \lesssim M \lesssim 8 M_{\odot}$ stars will explode as one (e.g., Maoz 2008), which suggests that the evolution leading to SN Ia production cannot be "fragile."

In this paper, we propose that the SN Ia rate has a strong dependence on stellar metallicity and examine the potential observational signatures in order to test the overall sign of this effect. We construct a simple model that examines the SN Ia rate in galaxies, as a function of galaxy mass, age, and metallicity, and in the universe at large. This is largely motivated by the measurements of the nearby SN Ia rates reported by the Lick Observatory SN Search (LOSS: Leaman et al. 2011; Li et al. 2011a, 2011b). These measurements and their interpretation are discussed in Section 2.

We present our model in Section 3, accounting first for a dependency of the SN Ia rate on galaxy mass and age alone. In Section 4, we expand upon our model to incorporate this possible effect of metallicity in regulating the SN Ia rate and discuss a variety of implications and competing effects, such as the dependence of stellar radius on $Z$. We extend this in Section 5 into a treatment of the cosmic SN Ia rate, addressing "prompt" and "delayed" SNe. In Section 6, we discuss methods to discern 


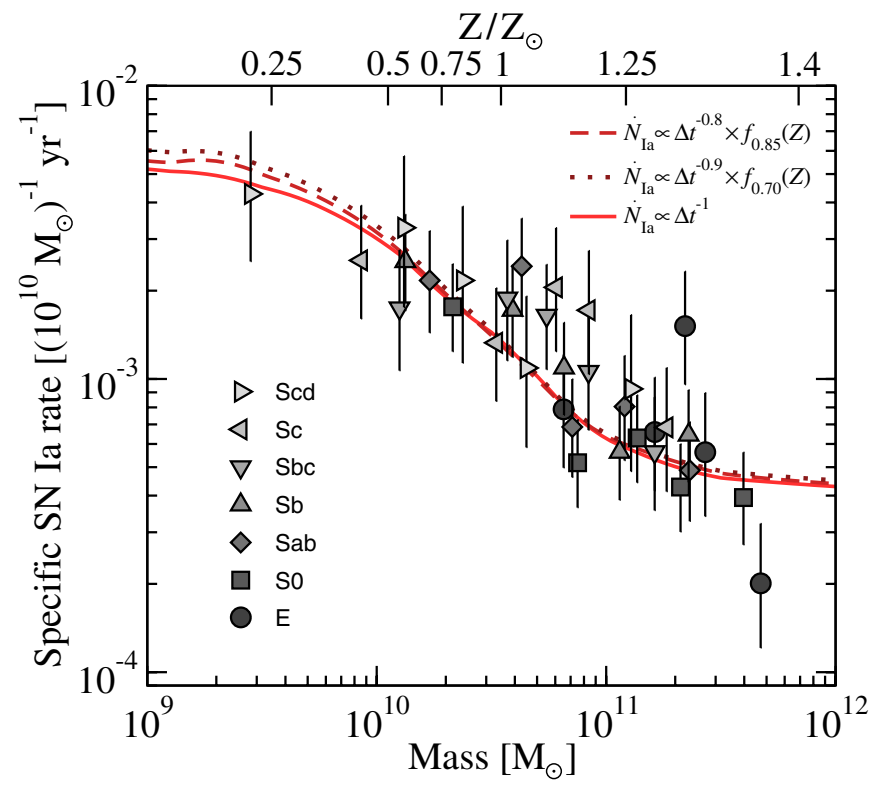

Figure 1. The specific rate of Type Ia supernovae vs. host galaxy mass. Shown are data from LOSS for galaxies grouped by Hubble type (Li et al. 2011a). Our models are also displayed, which assume either a $\Delta t^{-1}$ delay time distribution alone (solid line) or an additional dependence on stellar metallicity (dashed, dotted lines; see text).

(A color version of this figure is available in the online journal.)

the role of metallicity, including the differential examination of $\mathrm{SNe}$ Ia host galaxies, the hosts of high- $z \mathrm{SNe}$, galactic chemical evolution, $\mathrm{SNe}$ in galactic halos, and super-Chandrasekhar SNe, and effects on cosmological studies.

\section{THE TYPE Ia SUPERNOVA RATE IN GALAXIES}

Currently, the most complete and systematic search for nearby supernovae has been conducted over the past decade by the Lick Observatory SN Search, with results recently detailed in Leaman et al. (2011) and Li et al. (2011a, 2011b). Here, we briefly discuss the implications of the LOSS findings for our present study. Of particular interest are the results pertaining to $\mathrm{SNe}$ Ia.

In Figure 1, we display the specific $\mathrm{SN}$ Ia rate (rate per unit mass) versus galaxy mass as measured by LOSS (Li et al. 2011a). One is first struck by the steep dependence of this specific rate on galaxy mass. This variation of over an order of magnitude demands a physical explanation. The cause should be distinct from the origin of a similar pattern seen in the specific core-collapse supernova rate by LOSS, which likely arises mainly from the dependence of the specific star formation rate (SFR) on galaxy mass (Li et al. 2011a).

It is important to note that we also see that at a fixed mass, the SN Ia rate does not vary greatly between galaxies of different Hubble type. This suggests that by examining a large set of galaxies, one can arrive at the global behavior of SNe Ia. For our later use, we proceed to translate the LOSS measured specific $\mathrm{SN}$ Ia rates in galaxies of various Hubble types from a function of galactic mass into one of galactic metallicity. To do this, we convert between galactic mass and median metallicity using the relation derived from Sloan Digital Sky Survey (SDSS) data in Gallazzi et al. (2005), as shown by the upper axis of Figure 1. This technique effectively averages over a large representative galaxy population similar to that sampled by LOSS.

\section{A SIMPLE GALACTIC RATE MODEL}

We first attempt to explain the rate variations in galaxies of different mass as being due to an age effect alone. Since there is a delay from stellar birth to SN Ia explosion, a galaxy's SN Ia rate depends upon the age of its WD population. This is typically quantified by an empirical or theoretical delay-time distribution (DTD), which results in an SN Ia rate that can be simply written as

$$
\dot{N}_{\mathrm{Ia}}(t)=\int_{t_{0}}^{t} d t^{\prime} \phi\left(t-t^{\prime}\right) \dot{\rho}_{*}\left(t^{\prime}\right),
$$

where $t_{0}$ is the age of the universe when SN Ia progenitor stars first formed and $\phi\left(t-t^{\prime}\right)$ is the DTD, which maps between the rate of star formation at time $t^{\prime}, \dot{\rho}_{*}\left(t^{\prime}\right)$, and the SN Ia rate at a later time $t=t^{\prime}+\Delta t$. Equation (1) can be used to calculate the expected SN Ia rate of an individual galaxy or the universe as a whole, given a properly normalized $\dot{\rho}_{*}(t)$ (for the cosmic SN Ia rate, we will use the SFR density). Recent studies have suggested that $\phi$ roughly takes a $\Delta t^{-1}=\left(t-t^{\prime}\right)^{-1}$ form (e.g., Totani et al. 2008; Maoz et al. 2010, 2011). The physics behind this relation remains unclear, although such a distribution may naturally result from binary mergers (see, e.g., Ruiter et al. 2009) or a single-degenerate scenario (Hachisu et al. 2008).

Gallazzi et al. (2005) also derive $r$-band light-weighted galaxy ages, which vary from $\sim 10^{9}$ yr at $10^{9} M_{\odot}$ to $\sim 10^{10}$ yr at $10^{12} M_{\odot}$ (see their Figure 8), using galactic models with an exponentially declining star formation history (SFH) from a time $t_{\text {form }}$ with subsequent random bursts. Ideally, one would have at hand the detailed history of star formation in every galaxy. This is understandably difficult to achieve with any certainty. Attempts have been made in this direction (e.g., Brandt et al. 2010; Maoz et al. 2011); however, using what amounts to an average over the galaxy population should be suitable for comparison with global rates.

If the Gallazzi et al. (2005) ages corresponded to a singleage stellar population at a given galactic mass, then deriving the expected SN Ia rate for a given DTD would be rather straightforward. For example, using a DTD for each galaxy of the form

$$
\phi(\Delta t)=\phi_{*} \Delta t_{\mathrm{Gyr}}^{-\gamma}
$$

with $t_{\mathrm{Gyr}}=t /(1 \mathrm{Gyr})$, and assuming that the entire galactic stellar mass, $M_{g}$, arose at a single time, $t_{g}$, in Equation (1) would lead to a galactic specific SN Ia rate at time $t$ of

$$
\frac{\dot{N}_{g}(t)}{M_{g}}=\phi\left(t_{\mathrm{Gyr}}-t_{g, \mathrm{Gyr}}\right)=\frac{\phi_{*}}{\left(t_{\mathrm{Gyr}}-t_{g, \mathrm{Gyr}}\right)^{\gamma}} .
$$

This description is incomplete though. First, the SN Ia rate currently reflects the galactic mass at the time of formation, as opposed to that measured today after stellar mass loss has occurred. We correct for this using the results of Bruzual \& Charlot (2003) for a Chabrier IMF (as used in the SDSS galaxy sample) by including a term of the form $M\left(t_{g}\right) / M(t)$.

Additionally, the ages are more accurately galactic averages, so that an assumption of instantaneous formation at $t_{g}$ will not properly reflect the effect of a DTD. To allow for a finite duration of star formation, we use a declining history of the form $e^{-t / \tau}$, with $\tau=1 \mathrm{Gyr}$, occurring since the time $t_{g}$ for each galaxy. We further make use of the $16 / 84 \%$ ranges in $\log t_{g}$ reported in Gallazzi et al. (2005) in order to weight the galaxy population with the DTD at fixed mass (rather than using only the median value). These should alleviate the effect of 
average ages by giving greater weight to the low-age tail of their derived distribution and by allowing for a non-negligible rate of star formation today, particularly for lower-mass galaxies, that is in rough agreement with the specific SFRs measured by Schiminovich et al. (2007).

This leaves the issue of the efficiency of converting a stellar population into SNe Ia (see, e.g., Maoz 2008). As we will discuss in Section 5, the DTD is also involved in shaping the cosmic SN Ia history, with $\phi_{*}$ again setting the overall normalization. Rather than attempting to incorporate theoretical models of the DTD (see, e.g., Greggio 2010; Meng et al. 2011), we use a DTD with a pure power law of the form $\Delta t^{-\gamma}$, with a lower cutoff $t_{c}$ to account for the minimum amount of time needed to produce $\mathrm{CO}$ WDs. Evidence for delay times as short as $\lesssim 100$ Myr has been reported from, e.g., the study of SN remnants in the Magellanic Clouds (Badenes et al. 2009; Maoz \& Badenes 2010), and we simply use $t_{c}=50 \mathrm{Myr}$ (see also the discussion in Section 5).

The results from this approach are shown as the solid line in Figure 1, where we have used a $\Delta t_{\mathrm{Gyr}}^{-1}$ DTD with $\phi_{*}=$ $1.4 \times 10^{-3}\left(10^{10} M_{\odot}\right)^{-1} \mathrm{yr}^{-1}$. A parameterization for this model is given in the Appendix. We see that the saturation in age at high masses results in a plateau, which should be a rather robust feature due to the relatively small scatter in estimated ages around $\lesssim 10$ Gyr in this range, while the decrease in age at lower mass results in a rise in the SN Ia rate. Overall, this simplified model agrees rather well with the LOSS data. Recently, Graur \& Maoz (2013) followed the above prescription to compare with rates from a sample of SDSS SN Ia hosts, finding general agreement with our result.

\section{INCORPORATING METALLICITY DEPENDENCE}

Historically, studies have focused on deriving the DTD without taking into account the possible effects of stellar metallicity on the SN Ia rate in a galaxy. If there is no such effect, then what we have done above would be sufficient. As we show next, this assumption may prevent a determination of the actual DTD, and hence its astrophysical origins. We distinguish here between metallicity effects as primary (those involved in the rate of explosions) and secondary (those affecting the detailed properties of individual explosions; e.g., Timmes et al. 2003), with our interest being in the former.

We now examine a plausible scenario for including an SN Ia rate that varies with stellar metallicity. We propose that this arises from the effect of metallicity on the WDs produced (near the end of this section we discuss other possibilities). In general, it is expected that, for the same initial stellar mass, the WD from a star of lower metallicity should be more massive. This may be due to decreased mass loss and/or opacity resulting in hotter burning over the lifetime of the star (e.g., Umeda et al. 1999; Willson 2000; Marigo \& Girardi 2007; Meng et al. 2008). The simplest interpretation of this is that it should be easier to reach the requisite Chandrasekhar mass for explosion through the addition of mass via binary evolution or a double WD merger.

To obtain a semi-quantitative estimate of the resulting change in the SN Ia rate with metallicity, we must consider the effect of a varying WD mass over the range of metallicities for the galaxies in the LOSS sample. One may hope for guidance from the initial-final WD mass relation determined from young star clusters. However, the clusters for which detailed studies are possible are nearby and formed recently, which necessarily limits them to single, approximately solar metallicity stars (e.g., Kalirai et al. 2008; Williams et al. 2009). We utilize the theoretical results of Umeda et al. (1999) in combination with the empirical metallicity estimates in Gallazzi et al. (2005). By decreasing the initial stellar metallicity from $Z=0.03$ to 0.004 , roughly the range spanned in Figure 1, Umeda et al. (1999) determined that an additional $\sim 0.05-0.15 M_{\odot}$ is added to the CO remnant at fixed initial mass (see their Figure 6).

Figure 8 of Umeda et al. (1999) displays the relative number of SN Ia progenitors obtained from their stellar evolution model as a function of metallicity after integrating over a Salpeter IMF from a lower initial stellar mass (which varies with $Z$ ) corresponding to a fixed final WD mass to an upper mass at which point $\mathrm{ONeMg}$ WDs were expected to be produced (varying with $Z$ from $\sim 7$ to $8.5 M_{\odot}$ ). Using a threshold WD mass of $0.85 M_{\odot}$ yields a dependence on the rate with metallicity that can be approximately parameterized as

$$
N_{\mathrm{Ia}}(Z) \propto(Z+0.003)^{-0.5}
$$

over the range $Z=0.004-0.03$. Using a lower threshold mass of $0.7 M_{\odot}$ yields a slightly weaker dependence, due to the larger mass range of approximately

$$
N_{\mathrm{Ia}}(Z) \propto(Z+0.0015)^{-0.3} .
$$

To derive galactic rates, we again use an SN Ia rate for each galaxy $\propto \Delta t^{-\gamma}$ and scale directly to the Umeda et al. (1999) results, normalizing these relations to unity at $Z=0.025$, the metallicity of a characteristic $\sim 10^{11} M_{\odot}$ galaxy in the LOSS sample. Assuming $Z_{g}$ and $t_{g}$ to be separable, we use the $16 / 84 \%$ ranges in $\log Z_{g}$ from Gallazzi et al. (2005) to again weight the galaxy distribution at fixed mass and introduce an overall term to account for the effect of metallicity in Equation (1), either $f_{0.85}(Z)$ or $f_{0.70}(Z)$.

The specific SN Ia rates resulting from using the two metallicity scalings are shown in Figure 1 . For the $f_{0.85}$ model, $\phi_{*}=1.1 \times 10^{-3}\left(10^{10} M_{\odot}\right)^{-1} \mathrm{yr}^{-1}$ with $\Delta t_{\mathrm{Gyr}}^{-0.8}$ (dashed line), while the $f_{0.70}$ model has $\phi_{*}=1.3 \times 10^{-3}\left(10^{10} M_{\odot}\right)^{-1} \mathrm{yr}^{-1}$ and $\Delta t_{\mathrm{Gyr}}^{-0.9}$ (dotted line). After accounting for the weaker effects of the DTDs used, the rate does indeed rise more steeply at lower galactic masses than by taking into account age alone. This can be interpreted as a relative change in efficiency, an effect at the factor of $\sim 2$ level over the mass range of Figure 1 for the $f_{0.85}$ model and slightly less for the $f_{0.70}$ case.

Because galaxy mass is strongly correlated with both age and metallicity, it is inevitable that the models are relatively degenerate and that inferences about the DTD from galaxy populations may err without accounting for metallicity. Our simplified treatment of galactic star formation histories may somewhat underpredict $\mathrm{SN}$ Ia rates at intermediate masses. This may be refined through more detailed modeling, although, given the uncertainties in our inputs, we will not attempt to do so here. It is encouraging that such broad agreement with data is already seen using quite general assumptions.

We note here that the normalization of these models can be scaled up or down, although this will directly affect the normalization of the expected cosmic SN Ia rates through the DTD, as we will discuss in the following section. Note also that care should be taken in comparing these results, which examine the galaxy population as a whole, to those that distinguish between "passive" and "star-forming" galaxies (e.g., Sullivan et al. 2006).

The above is essentially based on an assumption of a singledegenerate scenario. While the full effects of metallicity on a 


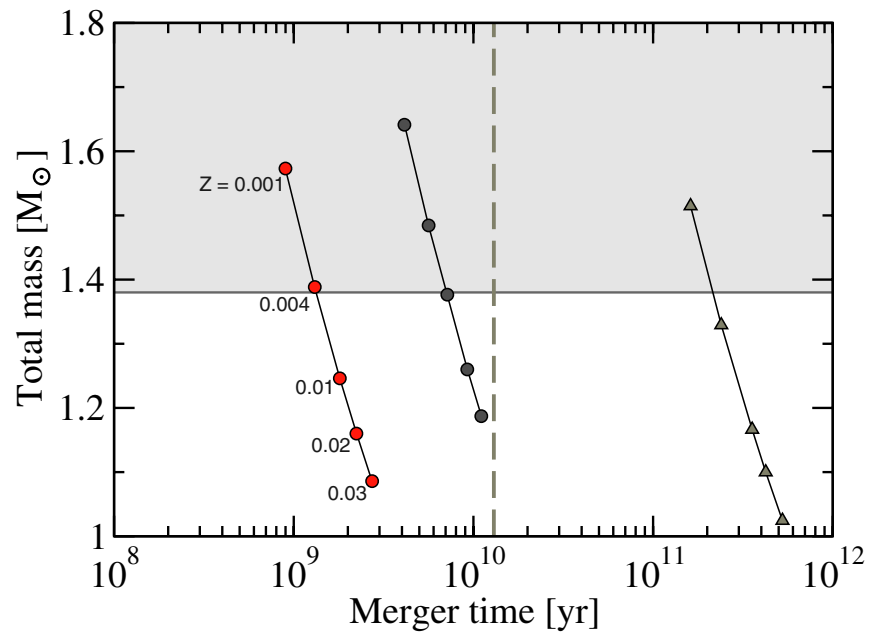

Figure 2. The total masses of known WD-WD systems along with their calculated merger times due to gravitational wave losses. Shown are the three systems from the collection in Nelemans et al. (2005) in which both WDs have masses exceeding $0.5 M_{\odot}$ : two with firm masses (circles) and one with only a lower limit on the mass of the secondary (triangles). Assuming these to have all resulted from stars with $Z \sim 0.02$, we show the "expected" total masses for a range of metallicities (as labeled) using the final masses derived in Umeda et al. (1999). The resulting merger times assume initial orbital separations as presently inferred for each.

(A color version of this figure is available in the online journal.)

double-degenerate scenario are likely more complicated, if we consider binaries with a uniform mass ratio distribution, then the number that will exceed the Chandrasekhar limit depends on metallicity as

$$
N_{\text {Ia }} \propto Z^{(x-1) b / a} \sim Z^{-0.4},
$$

where $x=2.35$ is the slope of the IMF, $a=0.5$ approximates the slope of the WD initial-final mass relation of Kalirai et al. (2008), and $b \simeq-0.08$ is the dependence of the final mass on metallicity, estimated from Umeda et al. (1999). The magnitude of the effect is very similar to the case already considered, so we do not repeat the calculations. This model does not include any effect of the higher implied masses on the rate of binary evolution or possible effects in triple systems based on the Kozai mechanism (Thompson 2011).

To illustrate the above effect, we begin with the three known double WD binaries from Nelemans et al. (2005) in which each component has a mass of at least $0.5 M_{\odot}$. These have primary/secondary masses of $0.71+0.55 M_{\odot}, 0.58+0.58 M_{\odot}$, and $0.51+>0.59 M_{\odot}$, the last being a single-lined system with only a lower limit for the secondary. We assume that each WD arose from a solar-metallicity star, and map from the WD masses to the initial stellar masses using the results of Umeda et al. (1999), mapping then to the WD masses calculated for these stellar masses at other metallicities. The $0.5 M_{\odot}$ cut allows a straightforward translation without regards to systems with lowmass He WDs, etc. Keeping the initial binary separations fixed, we calculate the merger time due to gravitational wave losses for each system (see, e.g., Thompson 2011). Figure 2 displays the effect on these systems using this prescription, where it is seen that the total masses of all three systems would have been pushed beyond the Chandrasekhar mass limit and the merger time would have been significantly reduced at lower metallicities.

There is hope for new tests to reduce the uncertainty in the overall effect of metallicity. For example, in observations of SN Ia host galaxies, we would expect the hosts of SN Ia to be slightly less metal-rich than the galaxy population as a whole for fixed galaxy mass. This effect would not be as marked as in the case of gamma-ray bursts (GRBs; see Stanek et al. 2006), since no hard upper metallicity threshold prohibiting the production of an SN Ia progenitor system is known to exist. An exception to this may be found at very high metallicity, as indicated by the abundance of He rather than CO WDs in the metal-rich cluster NGC 6791 (see Kilic et al. 2007)_-very massive, metal-rich galaxies may show an additional deficit of SNe Ia beyond that of our simple model. This may even be evident in the data at the high-mass end of Figure 1, although it is difficult to draw a strong conclusion at present.

We note that the model that we have used only results in a rather modest rate change with metallicity. It does not attempt to account for changes in the remnant mass that occur during the asymptotic giant branch phase of an isolated star (see, e.g., Vassiliadis \& Wood 1993; Bird \& Pinsonneault 2011; Renedo et al. 2010), which could result in a larger metallicity effect. Since both SN Ia scenarios require binary evolution at some step in their evolution, this model should be adequate in this respect. We also have not attempted to vary the binary fraction with stellar mass or metallicity. This is not yet well understood either theoretically or empirically (see, e.g., Mazeh et al. 2006), particularly in the mass range of the progenitors of SNe Ia.

In addition to a possible diminishment of stellar winds at low $Z$ (Kobayashi et al. 1998), another effect that may work in the opposite direction, particularly for single-degenerate scenarios, is the increased compactness of lower metallicity stars, which can limit interactions (de Mink et al. 2008a) and work toward a rate suppression. As a further complication, though, this aspect might also allow more of such stars to reach core helium burning and form a CO WD before mass transfer does occur (de Mink et al. 2008b). The net effect of varying stellar radii along with the number and masses of resulting WDs remains to be determined and should be considered in more detail at the level of binary evolution and in population synthesis modeling. All this suggests that substantial room for improvement exists on both the theory and observing fronts and an initial goal should be to determine the overall sign of the influence of metallicity on $\mathrm{SN}$ Ia rates.

\section{THE COSMIC TYPE Ia SUPERNOVA RATE}

We next examine the expectations for the cosmic rate of SNe Ia by again first considering a case without explicit metallicity dependence. We proceed by returning to Equation (1) with the comoving SFR density $\dot{\rho}_{*}(z)$ inferred up to $z \sim 8$, using the Yuksel et al. (2008) parameterization of the SFH,

$$
\dot{\rho}_{*}(z)=\dot{\rho}_{0}\left[(1+z)^{a \eta}+\left(\frac{1+z}{B}\right)^{b \eta}+\left(\frac{1+z}{C}\right)^{c \eta}\right]^{1 / \eta},
$$

where $a=3.4, b=-0.3$, and $c=-2$, with breaks at $z_{1}=1$ and $z_{2}=4$ corresponding to $B=\left(1+z_{1}\right)^{1-a / b} \simeq 5100$ and $C=\left(1+z_{1}\right)^{(b-a) / c}\left(1+z_{2}\right)^{1-b / c} \simeq 14$, which reflect the updated high- $z$ data from Kistler et al. (2009), and we use $\eta \simeq-10$ to smooth the transitions. The normalization is $\dot{\rho}_{0}=0.014 M_{\odot} \mathrm{yr}^{-1} \mathrm{Mpc}^{-3}$, which we have scaled down by a factor of 0.7 from the Salpeter IMF normalization of Hopkins $\&$ Beacom (2006) to be in better agreement with the galactic mass estimates in the previous sections. In Equation (1), we set $t_{0} \sim 0.4$ Gyr corresponding to $z \approx 10 .^{9}$

\footnotetext{
9 We use $\Omega_{\mathrm{m}}=0.3, \Omega_{\Lambda}=0.7$, and $H_{0}=70 \mathrm{~km} \mathrm{~s}^{-1} \mathrm{Mpc}^{-1}$ where needed, e.g., in converting $z \leftrightarrow t$ and in rescaling the data in Figure 3 using a common value of $H_{0}$
} 


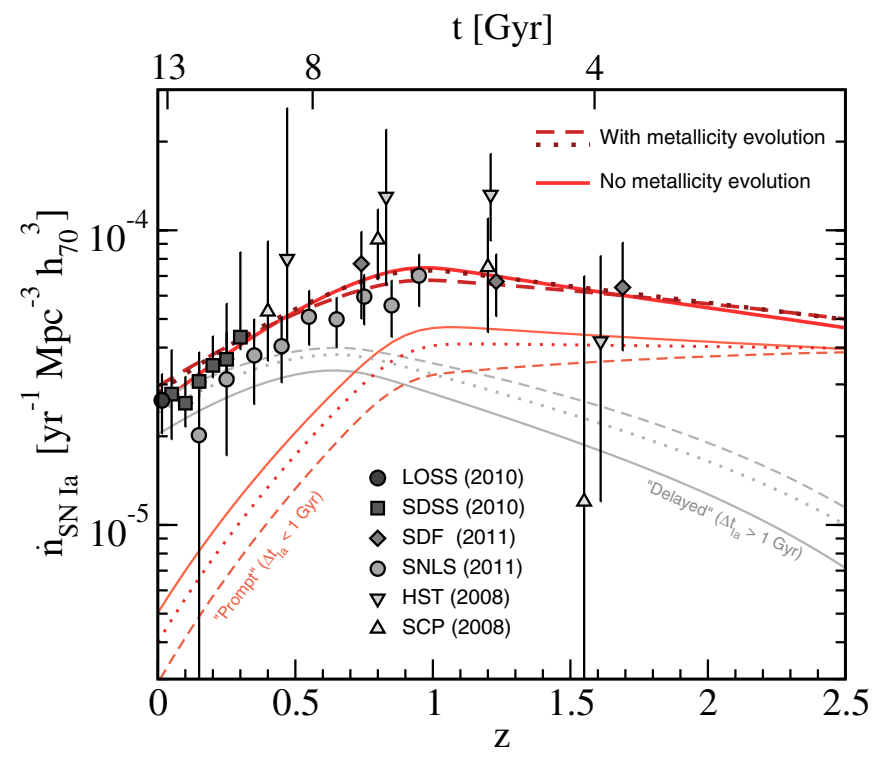

Figure 3. The cosmic rate of Type Ia supernovae. Shown are recent measurements from LOSS (Li et al. 2011a), SDSS (Dilday et al. 2010), SCP (Kuznetsova et al. 2008), HST (Dahlen et al. 2008), SNLS (González-Gaitán et al. 2011) and Subaru Deep Field (Graur et al. 2011). A model assuming only a fixed $\Delta t^{-1}$ delay time distribution and the cosmic SFR from Kistler et al. (2009; thick solid line) can be compared to our models incorporating metallicity dependence (see text), which use either a $\Delta t^{-0.9}$ DTD (thick dotted line) or $\Delta t^{-0.8}$ DTD (thick dashed line). The components of these models with delays from stellar birth to explosion of less than 1 Gyr ("prompt") and greater than 1 Gyr ("delayed") are also shown (thin lines; as labeled).

(A color version of this figure is available in the online journal.)

Since we must consider the shortest possible delay times in constructing the cosmic rate from the $\mathrm{SFH}$, we again assume a power law DTD with a cutoff at $t_{c}=50 \mathrm{Myr}$. We note that the results of Umeda et al. (1999; see also Siess 2007; Meng et al. 2008) suggest a maximum CO WD mass of $\sim 1.1 M_{\odot}$ that is nearly independent of metallicity. Since the effect of decreasing the metallicity is similar to increasing the stellar mass, we take this cutoff to be independent of $Z$ (and thus $z$ ) since the lifetimes of the stars giving the most massive CO WDs should be similar.

We use the $\Delta t_{\mathrm{Gyr}}^{-1}$ DTD and $\phi_{*}$ obtained by comparison to the LOSS data in Section 3, so that the resulting SN Ia rate history parameters are fixed, leading to the evolution shown in Figure 3 (thick solid line). To compare with prior results (e.g., Mannucci et al. 2006; Scannapieco \& Bildsten 2006; Sullivan et al. 2006), this history is broken down into the components with delay less than 1 Gyr (thin solid lines labeled as "prompt") and greater than 1 Gyr (labeled as "delayed"). We see that the "prompt" component is subdominant at $z=0$, in agreement with the rates in Figure 1. Altering either the form of the DTD or $t_{c}$ can make the "prompt" component relatively more or less important (see, e.g., Horiuchi \& Beacom 2010 for related discussion); however, this would in turn affect the specific SN Ia rate models in Sections 3 and 4.

Since the universe as whole had a lower metallicity in the past, a relative enhancement should also be effected in the cosmic SN Ia rate. As existing rate measurements average over the entirety of the galaxy population, this effect should not be dramatic at the present epoch, but, as for the specific rate, can be important in deriving the DTD. At low $z$, the gas-phase metallicity is typically higher than that of the stellar population (Gallazzi et al. 2005). The relation between galaxy mass and gas-phase metallicity is well determined at low $z$ (Tremonti et al. 2004) and has been measured to evolve at higher redshifts, so that the typical metallicity decreases by $\sim 0.15$ dex per $z$ up to at least $z \approx 2$ (e.g., Kewley \& Kobulnicky 2005; Savaglio et al. 2005; Erb et al. 2006; Maiolino et al. 2008). We use

$$
Z(z)=0.03 \times 10^{-0.15 z}
$$

to account for stars forming from gas that is increasingly metal poor at higher $z$, with a resulting change in rate arising through either the relation approximated by Equation (4) for the $\dot{n}_{0.85}$ model or Equation (5) for the $\dot{n}_{0.70}$ model, again normalizing each to unity at $Z=0.025$ to be consistent with our specific rate models. We also use the same values of $\phi_{*}$ and DTD slopes as in the corresponding specific rate models.

Figure 3 shows the resulting cosmic rates for the $\dot{n}_{0.85}$ (with $\Delta t_{\mathrm{Gyr}}^{-0.8}$; thick dashed line) and $\dot{n}_{0.70}\left(\Delta t_{\mathrm{Gyr}}^{-0.9}\right.$; thick dotted line $)$ models. Both models yield similar histories as the metallicityindependent case, with parameterizations for all three included in the Appendix. This is due to the relative increase of the rates with $z$ as compared to models with the same DTD without a metallicity enhancement. This is similar in spirit, but less dramatic, than the relative evolution likely due to stellar metallicity seen in the cosmic GRB rate (e.g., Kistler et al. 2008). Both models are also broken down by delay time in Figure 3 (thin dotted, dashed lines), which illustrates the underlying effect of altering the DTD.

As discussed for the specific SN Ia rate, there is again a degeneracy between altering the DTD and including the effect of metallicity, although not quite as strong. The fact that the metallicity effect works in the same direction as decreasing the index in the DTD in both cases, as seen in Figures 1 and 3, is something of a coincidence, owing to the fact that galaxy ages and metallicities both decrease with decreasing mass and the cosmic SFR rises with increasing $z$. This didn't have to be the case though. We thus reiterate that an estimate of one component must account for the other until this degeneracy is broken. It is possible to perform a more elaborate study by varying all the parameters involved (see, e.g., Horiuchi \& Beacom 2010 and Graur et al. 2011 for the metallicity-independent DTD); however, the qualitative effects of the models that we have considered are already sufficiently evident.

\section{DISCUSSION AND CONCLUSIONS}

The rate of SNe Ia should be affected at some level by the effects of metallicity on stellar evolution. There may be various complications involved, such as the largely unresolved effects of binary evolution, but our simple model for the effects of metallicity should be broadly relevant. There has been significant effort devoted to investigating SN Ia properties as a function of metallicity (e.g., Hamuy et al. 2000; Gallagher et al. 2008; Howell et al. 2009; Neill et al. 2009; Sullivan et al. 2010; Konishi et al. 2011). Since the properties of SN Ia have been observed to depend on metallicity, why not the rate?

The simple models that we have considered explain fairly well both the specific SN Ia rates measured in nearby galaxies by LOSS and the observed normalization and evolution of the cosmic SN Ia rate. An enhanced rate due to more massive WD remnants is in contrast to other effects that may be important during binary interactions, such as a decrease of stellar winds or radius. Future models in this area can utilize the framework presented here as a basis for comparison with data. Attempts to introduce the various effects discussed into population synthesis models are welcome. Since the full problem of solving the 
complete chain of events of binary evolution leading to an SN Ia is very difficult, a reasonable first step would be to determine empirically whether there is a net enhancement or suppression.

A low- $Z$ enhancement leads to an expectation of a relatively higher SN Ia rate in the outer regions of galaxies, for a given stellar population age and total mass, due to the lower average metallicity. It is thus important to take into account not just the integrated metallicity of the galaxy, but the value at the birthplace of the progenitor. Such a bias may already be seen in a number of cases in which an SN Ia occurred in the outer halo of a star-forming galaxy (Prieto et al. 2008; Khan et al. 2011). Using the location of the explosion as a proxy in such a differential study would be cleanest performed by considering "prompt" SNe Ia and/or small, more metallicity-homogeneous galaxies.

Further progress can certainly be made with data that can thus suitably break the degeneracy between decreasing metallicity and decreasing age. Evidence in this direction has been found in a comparison of SN Ia host galaxies in SDSS by Cooper et al. (2009), who found that SNe Ia in blue, star-forming hosts have a preference for lower-density environments, which they interpreted as being the effect of lower gas-phase metallicities. Non-targeted SN searches are useful in this regard, such as ROTSE-IIIb, which found an excess of dwarf hosts in their SN Ia sample (Quimby et al. 2012).

New efforts to discover ever-higher-redshift SNe Ia are also necessarily probing a regime where the intrinsic metallicity is lower. Such surveys have recently uncovered two $\mathrm{SNe}$ Ia at $z \simeq 1.55$ with very-low $Z$ hosts, one with $12+\log (\mathrm{O} / \mathrm{H})=$ $8.12_{-0.10}^{+0.09}$ (Frederiksen et al. 2012) and another with the rather low $12+\log (\mathrm{O} / \mathrm{H})<8.0$ (Frederiksen et al. 2013). Taking these as upper limits on the $\mathrm{SN}$ progenitor stellar $Z$ places pressure on models with a metallicity floor. The model of Kobayashi et al. (1998) in particular, with a minimum metallicity of $\sim 0.1 Z_{\odot}$, predicts a negligible number of SNe Ia at $z \gtrsim 1$. In contrast, the continued observation of high- $z$ supernovae, such as the recent $z=1.914$ event (Jones et al. 2013), is expected in the picture we put forward. Since the evolution with $z$ of the $M-Z$ relation is seen to proceed more rapidly at low masses (Zahid et al. 2013), we would thus anticipate relatively more discoveries of such prompt $\mathrm{SNe}$ in low-mass hosts, which as discussed above are favored for relative rate analyses.

Additionally, observations of Milky Way dwarf spheroidal galaxies have revealed decreasing values of $[\alpha / \mathrm{Fe}]$ with increasing $[\mathrm{Fe} / \mathrm{H}]$, indicating the influence of SNe Ia down to metallicities of $[\mathrm{Fe} / \mathrm{H}] \approx-2.5$ (Kirby et al. 2011). Since model fits to these measurements are naturally sensitive to the SN Ia rate over a range of metallicities, we urge exploration of the implications of an increased rate at low $Z$, including superChandrasekhar mergers, on galactic chemical evolution.

As previously mentioned, the results of Umeda et al. (1999) indicate that the maximum $\mathrm{CO}$ WD mass remains close to $\sim 1.1 M_{\odot}$ over a wide range of metallicities. If this is true and binary evolution effects are neglected, then we would expect the relative rates of super-Chandrasekhar SNe Ia arising from mergers to increase with lower metallicity in proportion to the normal SNe Ia due to the power law form of the IMF (note that instabilities prohibit the necessary growth of even rapidly-rotating single WDs; Piro 2008). However, recent observations of host galaxies may indicate an even stronger preference for low-metallicity hosts for super-Chandrasekhar SNe Ia (e.g., Taubenberger et al. 2011; Childress et al. 2011).
Moreover, the maximum mass resulting from a merger under these assumptions is $\sim 2.2 M_{\odot}$, below the $2.4 \pm 0.2 M_{\odot}$ total mass inferred from SN 2007if (Scalzo et al. 2010).

An explanation for both effects may arise from binary evolution. To achieve a higher total merger mass without resorting to an $\mathrm{ONeMg} \mathrm{WD}$, at least one WD should gain mass while maintaining a $\mathrm{CO}$ composition. If the effect of inhibiting single degenerate $\mathrm{SN}$ Ia production at $Z \lesssim 0.1 Z_{\odot}$ (Kobayashi et al. 1998; Kobayashi \& Nomoto 2009) does hold, then the primary WD may instead be pushed close to, but not above, the threshold for explosion, so that the rate of massive mergers is further enhanced. The end state of the secondary resulting in a massive CO WD could then lead to a merger with a total mass upward of $\sim 2.5 M_{\odot}$.

If, contrary to Umeda et al. (1999), the CO WD mass limit actually increases modestly for lower metallicities, then the rate of such extreme super-Chandrasekhar mergers rises dramatically at lower metallicities without a need to turn to binary evolution for a solution. This is due to the presence of the threshold in reaching the requisite total merger mass, which would lead to a large relative difference between low/high- $Z$ galaxies. The stars giving rise to these massive WDs would also evolve more rapidly and could thus lead to "prompter" explosions in low- $Z$ environments. Whether this scenario occurs is a question that remains for stellar evolutionary modeling and observations of host galaxies. We note that elevated rates of other transients involving a WD and dependent upon the mass (e.g., Thompson et al. 2009) could also be expected.

In the category of interesting but more anecdotal evidence that low metallicity might be of significance for SNe Ia, Tovmassian et al. (2010) recently presented strong evidence that SBS1150+599A, a close binary star inside a metal-poor, Galactic halo planetary nebula PN G135.9+55.9 consists of two WDs that will merge within a Hubble time. The estimated total mass of the binary is very close to the Chandrasekhar limit, making it a likely SN Ia progenitor.

It is also interesting to note that the normalized rate of PNe in elliptical galaxies (Buzzoni et al. 2006) shows a very similar trend with metallicity to that discussed in our Figure 1. Indeed, their Figures 11 and 12 show about 10 times fewer PNe per unit luminosity in metal-rich, massive ellipticals compared to metalpoor, low-mass ellipticals. The mapping between $\mathrm{PN}$ production and SN Ia explosion is of course uncertain; however, both involve the production of a WD, and Buzzoni et al. (2006) attribute finding fewer PNe in more metal rich ellipticals to a dependence of the initial-to-final mass relation on metallicity.

Substantial observational progress has been made in the study of $\mathrm{SNe}$ Ia in the last decade and new data can be expected to better determine the extent to which metallicity affects the SN Ia rate. As discussed above, the possible directions include detailed measurements of rates within galaxies to examine the $Z$ dimension. Improved measurements of the cosmic SN Ia rate, in combination with independent determinations of the DTD at fixed $Z$, can examine whether the rate is larger than otherwise expected. In addition to these, if the intrinsic properties of SNe Ia vary with metallicity, then evolution in the Type Ia luminosity function can complicate cosmological determinations (Riess \& Livio 2006), which in the case presented here would be more pronounced as the lower- $Z$ component becomes further enhanced at higher redshifts. This places added emphasis on the importance of determining the net effect of metallicity on the SN Ia rate, and if a null result is eventually established, how the various effects discussed here could conspire in such a way. 
Table 1

Parameters Used in the Fits of Our Three Cosmic SN Ia Rate Scenarios

\begin{tabular}{lcccccrrr}
\hline \hline Model & $\begin{array}{c}\dot{n}_{0} \\
\left(\mathrm{yr}^{-1} \mathrm{Mpc}^{-3}\right)\end{array}$ & $a$ & $b$ & $c$ & $z_{1}$ & $z_{2}$ & $B$ & $C$ \\
\hline$Z$-free & $2.5 \times 10^{-5}$ & 1.8 & -0.8 & -2.3 & 0.9 & 2.9 & 8.1 & 5.0 \\
$\dot{n}_{0.85}$ & $2.9 \times 10^{-5}$ & 1.4 & -0.5 & -2.0 & 0.9 & 2.9 & 11.5 & 5.1 \\
$\dot{n}_{0.70}$ & $2.8 \times 10^{-5}$ & 1.6 & -0.7 & -2.0 & 0.9 & 3.0 & 8.2 & 5.2 \\
\hline
\end{tabular}

We thank John Beacom, Jonathan Bird, Shunsaku Horiuchi, Rubab Khan, Marc Pinsonneault, and Hasan Yuksel for helpful discussions, Weidong Li for providing us with the data in Figure 1, and the referee for helpful comments. M.D.K. acknowledges support provided by NASA through the Einstein Fellowship Program, grant PF0-110074. K.Z.S., C.S.K. and T.A.T. are supported in part by NSF grant AST-0908816. J.L.P. acknowledges support from NASA through Hubble Fellowship grant HF-51261.01-A awarded by the STScI, which is operated by AURA, Inc. for NASA, under contract NAS 5-26555. T.A.T. is supported in part by an Alfred P. Sloan Foundation Fellowship.

\section{APPENDIX}

The models that we have discussed are the result of combining several unrelated functions and thus are not necessarily amenable to convenient parameterization. Nonetheless, we find that a sigmoid function provides an adequate fit to our metallicity-independent model of the specific SN Ia rate, $\zeta_{\text {Ia }}$, with

$$
\begin{aligned}
& \frac{\zeta_{\mathrm{Ia}}(M)}{\left(10^{10} M_{\odot}\right)^{-1} \mathrm{yr}^{-1}} \\
& \quad=\alpha\left[1+\exp \left(\frac{\log \left(M / M_{\odot}\right)-M_{*}}{\omega}\right)\right]^{-1}+\beta,
\end{aligned}
$$

where $\alpha=5 \times 10^{-3}, \beta=4.2 \times 10^{-4}, M_{*}=10$, and $\omega=0.33$ agrees with the model to within $<10 \%$ over the mass range displayed in Figure 1. The metallicity-dependent models can be fit with similar parameters.

Using the smoothly-broken piecewise form of Equation (7), with $\dot{\rho}_{0}$ replaced by $\dot{n}_{0}$, our cosmic rate models can be fit to within a few percent over the range $z=0-4$. The parameters used for the metallicity-independent model and the metallicitydependent $\dot{n}_{0.85}$ and $\dot{n}_{0.70}$ models are given below in Table 1. All three use $\eta \simeq-10$ to smooth the transitions.

\section{REFERENCES}

Badenes, C., Harris, J., Zaritsky, D., \& Prieto, J. L. 2009, ApJ, 700, 727

Bird, J. C., \& Pinsonneault, M. H. 2011, ApJ, 733, 81

Brandt, T. D., Tojeiro, R., Aubourg, E., et al. 2010, AJ, 140, 804

Bruzual, G., \& Charlot, S. 2003, MNRAS, 344, 1000

Buzzoni, A., Arnaboldi, M., \& Corradi, R. L. M. 2006, MNRAS, 368, 877

Childress, M., Aldering, G., Aragon, C., et al. 2011, ApJ, 733, 3

Cooper, M. C., Newman, J. A., \& Yan, R. 2009, ApJ, 704, 687

Dahlen, T., Strolger, L. G., \& Riess, A. G. 2008, ApJ, 681, 462

Dan, M., Rosswog, S., Guillochon, J., \& Ramirez-Ruiz, E. 2012, MNRAS, 422, 2417

de Mink, S. E., Cottaar, M., \& Pols, O. R. 2008a, in AIP Conf. Proc. 990, First Stars III: First Stars II Conference, ed. B. W. O'Shea, A. Heger, \& T. Abel (Melville, NY: AIP), 217

de Mink, S. E., Pols, O. R., \& Yoon, S. C. 2008b, in AIP Conf. Proc., 990, First Stars III: First Stars II Conference, ed. B. W. O’Shea, A. Heger, \& T. Abel (Melville, NY: AIP), 230

Dilday, B., Smith, M., Bassett, B., et al. 2010, ApJ, 713, 1026
Erb, D. K., Shapley, A. E., Pettini, M., et al. 2006, ApJ, 644, 813 Frederiksen, T. F., Graur, O., Hjorth, J., Maoz, D., \& Poznanski, D. 2013, ApJ, 768,166

Frederiksen, T. F., Hjorth, J., Maund, J. R., et al. 2012, ApJ, 760, 125

Fryer, C. L., Ruiter, A. J., Belczynski, K., et al. 2010, ApJ, 725, 296

Gallagher, J. S., Garnavich, P. M., Caldwell, N., et al. 2008, ApJ, 685, 752

Gallazzi, A., Charlot, S., Brinchmann, J., White, S. D. M., \& Tremonti, C. A. 2005, MNRAS, 362, 41

Gilfanov, M., \& Bogdan, A. 2010, Natur, 463, 924

González-Gaitán, S., Perrett, K., Sullivan, M., et al. 2011, ApJ, 727, 107

Graur, O., \& Maoz, D. 2013, MNRAS, 430, 1746

Graur, O., Poznanski, D., Maoz, D., et al. 2011, MNRAS, 417, 916

Greggio, L. 2010, MNRAS, 406, 22

Hachisu, I., Kato, M., \& Nomoto, K. 2008, ApJL, 683, L127

Hachisu, I., Kato, M., \& Nomoto, K. 2010, ApJL, 724, L212

Hamuy, M., Trager, S. C., Pinto, P. A., et al. 2000, AJ, 120, 1479

Hopkins, A. M., \& Beacom, J. F. 2006, ApJ, 651, 142

Horiuchi, S., \& Beacom, J. F. 2010, ApJ, 723, 329

Howell, D. A., Sullivan, M., Brown, E. F., et al. 2009, ApJ, 691, 661

Iben, I. J., \& Tutukov, A. V. 1984, ApJS, 54, 335

Jones, D. O., Rodney, S. A., Riess, A. G., et al. 2013, arXiv:1304.0768

Kalirai, J. S., Hansen, B. M. S., Kelson, D. D., et al. 2008, ApJ, 676, 594

Kasen, D., Röpke, F. K., \& Woosley, S. E. 2009, Natur, 460, 869

Kewley, L., \& Kobulnicky, H. A. 2005, in Starburst: From 30 Doradus to Lyman Break Galaxies, ed. R. de Grijs \& R. M. Gonzalez Delgado (Dordrecht: Springer), 307

Khan, R., Prieto, J. L., Pojmański, G., et al. 2011, ApJ, 726, 106

Kilic, M., Stanek, K. Z., \& Pinsonneault, M. H. 2007, ApJ, 671, 761

Kirby, E. N., Cohen, J. G., Smith, G. H., et al. 2011, ApJ, 727, 79

Kistler, M. D., Yüksel, H., Beacom, J. F., Hopkins, A. M., \& Wyithe, J. S. B. 2009, ApJL, 705, L104

Kistler, M. D., Yüksel, H., Beacom, J. F., \& Stanek, K. Z. 2008, ApJL, 673, L119

Kobayashi, C., \& Nomoto, K. 2009, ApJ, 707, 1466

Kobayashi, C., Tsujimoto, T., Nomoto, K., Hachisu, I., \& Kato, M. 1998, ApJL, 503, L155

Konishi, K., et al. 2011, arXiv:1101.4269

Kuznetsova, N., et al. 2008, ApJ, 673, 981

Langer, N., Deutschmann, A., Wellstein, S., \& Hoeflich, P. 2000, A\&A, 362, 1046

Leaman, J., Li, W., Chornock, R., \& Filippenko, A. V. 2011, MNRAS, 412, 1419

Leonard, D. C. 2007, ApJ, 670, 1275

Li, W., Chornock, R., Leaman, J., et al. 2011a, MNRAS, 412, 1473

Li, W., Leaman, J., Chornock, R., et al. 2011b, MNRAS, 412, 1441

Maiolino, R., Nagao, T., Grazian, A., et al. 2008, A\&A, 488, 463

Mannucci, F., Della Valle, M., \& Panagia, N. 2006, MNRAS, 370, 773

Maoz, D. 2008, MNRAS, 384, 267

Maoz, D., \& Badenes, C. 2010, MNRAS, 407, 1314

Maoz, D., Mannucci, F., Li, W., et al. 2011, MNRAS, 412, 1508

Maoz, D., Sharon, K., \& Gal-Yam, A. 2010, ApJ, 722, 1879

Marigo, P., \& Girardi, L. 2007, A\&A, 469, 239

Mazeh, T., Tamuz, O., \& North, P. 2006, MNRAS, 367, 1531

Meng, X., Chen, X., \& Han, Z. 2008, A\&A, 487, 625

Meng, X., Li, Z., \& Yang, W. 2011, PASJ, 63, L31

Neill, J. D., Sullivan, M., Howell, D. A., et al. 2009, ApJ, 707, 1449

Nelemans, G., Napiwotzki, R., Karl, C., et al. 2005, A\&A, 440, 1087

Pakmor, R., Kromer, M., Röpke, F. K., et al. 2010, Natur, 463, 61

Pakmor, R., Kromer, M., Taubenberger, S., et al. 2012, ApJL, 747, L10

Piro, A. L. 2008, ApJ, 679, 616

Prieto, J. L., Stanek, K. Z., \& Beacom, J. F. 2008, ApJ, 673, 999

Quimby, R. M., Yuan, F., Akerlof, C., Wheeler, J. C., \& Warren, M. S. 2012, AJ, 144,177

Renedo, I., Althaus, L. G., Miller Bertolami, M. M., et al. 2010, ApJ, 717, 183

Riess, A. G., \& Livio, M. 2006, ApJ, 648, 884

Ruiter, A. J., Belczynski, K., \& Fryer, C. L. 2009, ApJ, 699, 2026 
Savaglio, S., Glazebrook, K., Le Borgne, D., et al. 2005, ApJ, 635, 260 Scalzo, R. A., Aldering, G., Antilogus, P., et al. 2010, ApJ, 713, 1073 Scannapieco, E., \& Bildsten, L. 2006, ApJL, 629, L85

Schiminovich, D., Wyder, T. K., Martin, D. C., et al. 2007, ApJS, 173, 315

Shen, K. J., Bildsten, L., Kasen, D., \& Quataert, E. 2012, ApJ, 748, 35

Siess, L. 2007, A\&A, 476, 893

Simon, J. D., Gal-Yam, A., Gnat, O., et al. 2009, ApJ, 702, 1157

Stanek, K. Z., Gnedin, O. Y., Beacom, J. F., et al. 2006, AcA, 56, 333

Sullivan, M., Conley, A., Howell, D. A., et al. 2010, MNRAS, 406, 782

Sullivan, M., Le Borgne, D., Pritchet, C. J., et al. 2006, ApJ, 648, 868

Taubenberger, S., Benetti, S., Childress, M., et al. 2011, MNRAS, 412, 2735

Thompson, T. A. 2011, ApJ, 741, 82

Thompson, T. A., Kistler, M. D., \& Stanek, K. Z. 2009, arXiv:0912.0009

Timmes, F. X., Brown, E. F., \& Truran, J. W. 2003, ApJL, 590, L83
Totani, T., Morokuma, T., Oda, T., Doi, M., \& Yasuda, N. 2008, PASJ, 60,1327

Tovmassian, G., Yungelson, L., Rauch, T., et al. 2010, ApJ, 714, 178

Tremonti, C. A., Heckman, T. M., Kauffmann, G., et al. 2004, ApJ, 613, 898

Umeda, H., Nomoto, K., Yamaoka, H., \& Wanajo, S. 1999, ApJ, 513, 861

Vassiliadis, E., \& Wood, P. R. 1993, ApJ, 413, 641

Webbink, R. F. 1984, ApJ, 277, 355

Whelan, J., \& Iben, I. 1973, ApJ, 186, 1007

Williams, K. A., Bolte, M., \& Koester, D. 2009, ApJ, 693, 355

Willson, L. A. 2000, ARA\&A, 38, 573

Yuksel, H., Kistler, M. D., Beacom, J. F., \& Hopkins, A. M. 2008, ApJL, 683, L5

Zahid, J., Geller, M., Kewley, L., et al. 2013, arXiv:1303.5987

Zhu, C., Chang, P., van Kerkwijk, M., \& Wadsley, J. 2013, ApJ, 767, 164 